\title{
Rechtzeitige Prüfung ratsam
}

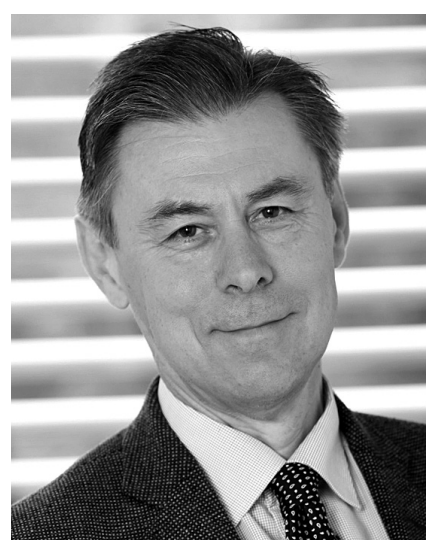

VON RALF KLASSMANN

Ralf Klaßmann ist Wirtschaftsprüfer und Steuerberater bei der BDO AG Wirtschaftsprüfungsgesellschaft. Er ist Sprecher der Standortleitung Köln und Leiter des Branchencenters Gesundheit und Soziales.

ralf.klassmann@bdo.de

\author{
Viele steuerbegünstigte soziale Organisationen \\ und Unternehmen sind unsicher, ob sie künftig \\ ihre Bilanzen oder Gewinn- und Verlustrechnungen \\ elektronisch an die Finanzverwaltung übermitteln \\ müssen. Ein Prüfschema kann Gewissheit bringen.
}

Das Steuerrecht schreibt - eigentlich verpflichtend - für Wirtschaftsjahre, die nach dem 31. Dezember 2011 beginnen, allen bilanzierenden Steuerpflichtigen eine Übermittlung entweder der (Handels-) Bilanz und der (zugehörigen) Gewinn- und Verlustrechnung nebst steuerlicher Überleitungsrechnung oder unmittelbar einer Steuerbilanz elektronisch nach amtlich vorgeschriebenem Datensatz (Taxonomie) durch Datenfernübertragung in Form von XBRLDatensätzen an das zuständige Finanzamt vor.

Von dieser Verpflichtung sind steuerbegünstigte Körperschaften beispielsweise in der Rechtsform des eingetragenen Vereins, der Stiftung oder der gGmbH grundsätzlich nicht ausgenommen. Sie werden vielmehr so behandelt wie jeder andere bilanzierende Steuerpflichtige.

Diese Verpflichtung würde für eine steuerbegünstigte Körperschaft in aller Regel erhebliche organisatorische Veränderungen im Rechnungswesen und in der EDV erforderlich machen. Denn auf die gesetzlichen Vorgaben des amtlich vorgeschriebenen Datensatzes (Taxonomie) und der Datenfernübertragung in Form von XBRL-Datensätzen ist so gut wie keine steuerbegünstigte Körperschaft vorbereitet.

Betroffen ist eine steuerbegünstigte Körperschaft - wie jeder andere Steuerpflichtige auch - aber nur, wenn sie bilanzierungspflichtig ist, wobei die Bilanzierungspflicht auf handelsrechtlichen Bestimmungen, beispielsweise des Handelsgesetzbuches oder der Pfle- gebuchführungsverordnung, oder auf steuerlichen Vorschriften (der Abgabenordnung) beruhen kann.

\section{Körperschaften ohne steuerpflichtige wirtschaftliche Geschäftsbetriebe}

Besteht Bilanzierungspflicht, kommt u. U. gleichfalls eine völlige Freistellung von den E-Bilanz-Vorgaben in Betracht, und zwar dann, wenn eine steuerbegünstigte Körperschaft nur ihre ideellen steuerbegünstigten Zwecke in sogenannten Zweckbetrieben beispielsweise in der Jugend-, Alten- oder Behindertenhilfe oder im Gesundheitswesen oder im Wohlfahrtswesen verfolgt. Dass daneben auch vermögensverwaltende Tätigkeiten (Vermietung und Verpachtung oder Kapitalanlage) geschehen, ist unbeachtlich.

\section{Bilanzierungspflichtige Körperschaften mit steuerpflichtigen wirtschaftlichen Geschäftsbetrieben}

1. Die Übergangszeit von 2012 bis 2014: Besteht Bilanzierungspflicht, wird es vom Bundesfinanzministerium "zur Vermeidung unbilliger Härten « für eine Übergangszeit »nicht beanstandet«, wenn steuerbegünstigte, bilanzierungspflichtige Körperschaften die Inhalte der Bilanz und der Gewinn- und Verlustrechnung erstmals für Wirtschaftsjahre, die nach dem 31. Dezember 2014 beginnen, durch Datenfernübertragung übermitteln, 
also erstmals im Jahre 2016 für 2015. Für die Übergangszeit (2012 bis 2014) dürfen die Gewinnermittlungen wie bisher »in Papierform « abgegeben werden; elektronische XBRL-Datensätze und die Anwendung des amtlich vorgeschriebenen Datensatzes, der sogenannten »Taxonomie « werden nicht gefordert. In der Übergangszeit bleibt also auch bei diesen Körperschaften letztlich alles beim Alten; bei ihnen herrscht derzeit deswegen noch Ruhe. Gleichwohl stellt sich bei ihnen die Frage, was denn demnächst (erstmals in 2016 für 2015) bei der elektronischen Übermittlung im XBRL-Format unter Anwendung der Taxonomie konkret zu beachten ist.

2. Die Zeit ab 2015: Das Bundesfinanzministerium hat dazu Stellung genommen und durchaus erfreuliche Regelungen gefunden. Danach sind die Vorgaben zur E-Bilanz auch dann generell ohne Bedeutung, wenn steuerpflichtige wirtschaftliche Geschäftsbetriebe unterhalten werden, deren Gewinne bekanntlich der Körperschaftsteuer und Gewerbesteuer unterliegen, beispielsweise in der Form von Besucherbeköstigungen oder aktiven Werbetätigkeiten für Sponsoren. Dies gilt jedenfalls immer dann, wenn die sogenannte Besteuerungsgrenze von jährlich 35.000 Euro bezogen auf die Einnahmen (nicht: Gewinne!) aus diesen steuerpflichtigen Aktivitäten - gegebenenfalls einschließ lich Umsatzsteuer -, nicht überschritten wird, wobei aber zusätzlich vorausgesetzt wird, dass diese Körperschaften ihren (steuerpflichtigen) Gewinn durch Einnahmen-Überschuss-Rechnungen ermitteln, was fast alle betroffenen Körperschaften in der Vergangenheit gemacht haben. Sollten steuerpflichtige wirtschaftliche Geschäftsbetriebe unterhalten werden, deren Einnahmen über der Besteuerungsgrenze von jährlich 35.000 Euro (ggf. incl. Umsatzsteuer) liegen, und sollte aufgrund gesetzlicher Verpflichtungen eine Bilanz aufzustellen sein, beispielsweise nach den Vorgaben des Handelsgesetzbuches, sind die E-Bilanz-Spielregeln zwar grundsätzlich zu beachten. Tatsächlich ist dann aber verpflichtend nur ein Datensatz für den steuerpflichtigen Teilbereich zu übermitteln (sog. "Minimalanforderungen«).

Bei der Übermittlung dieser »Minimalanforderungen « ist im Übrigen eine Übermittlung der »formlosen Ge- winnermittlung für den partiell steuerpflichtigen Teilbereich « zulässig, wenn die steuerbegünstigte Körperschaft nur eine Gesamtbilanz aufstellt und den Gewinn des partiell steuerpflichtigen Teilbereichs in einer (außerbilanziellen) Nebenrechnung ermittelt, was in den weitaus meisten Fällen geschieht.

\section{Sonderproblem: Steuerliche Bilanzierungspflicht nach der Abgabenordnung}

$\mathrm{Zu}$ beachten ist allerdings noch, dass steuerbegünstigte Körperschaften mit ihren (eigentlich) steuerpflichtigen Aktivitäten häufig nicht nur die bereits angesprochene Besteuerungsgrenze überschreiten, sondern auch noch die »Grenzen des $\mathbb{} 141 \mathrm{AO}$ « und deshalb von ihrem zuständigen Finanzamt eine "gesonderte Aufforderung « zur steuerlichen Bilanzierung bekommen könnten. Sollte eine solche Aufforderung vorliegen, könnten die beschriebenen Erleichterungen bei der E-Bilanz möglicherweise nicht zur Anwendung kommen. Dies ist derzeit noch nicht eindeutig geklärt.

$\mathrm{Zu}$ den »Grenzen des $\mathbb{} 141 \mathrm{AO}$ « gehören Umsätze von mehr als 500.000 Euro im Kalenderjahr und ein Gewinn aus Gewerbebetrieb - also aus den steuerpflichtigen wirtschaftlichen Geschäftsbetrieben - von mehr als 50.000 Euro im Wirtschaftsjahr. Ist eine dieser Grenze überschritten, besteht eine Bilanzierungspflicht auch dann, wenn sich eine Buchführungspflicht nicht aus anderen Vorschriften, beispielsweise des Handelsgesetzbuches, ergibt.

Diese Verpflichtung ist aber erst nach einem entsprechenden Hinweis des zuständigen Finanzamtes zu beachten. Solange dieser Hinweis nicht erfolgt ist, tritt keine originäre steuerliche Bilanzierungspflicht ein.

Wenn also - wofür derzeit vieles spricht - solche Aufforderungen konsequent von den Finanzämtern unterbleiben, ist künftig die elektronische Übermittlung einer »formlosen Gewinnermittlung für den partiell steuerpflichtigen Teilbereich « ausreichend, und zwar als Einzelbetrag (zur Plausibilisierung mit der Steuererklärung). Ob die elektronische Übermittlung eines solchen Einzelbetrags auch dann ausreicht, wenn eine Aufforderung im

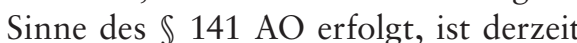

offen. Möglicherweise wird sich die Finanzverwaltung auch in diesen Fällen mit der elektronischen Übermittlung eines Einzelbetrags begnügen und die »eigentliche" Gewinnermittlung als Einnahmen-Überschuss-Rechnung nur »in Papierform« fordern.

\section{Schlussbemerkung: Prüfungsschema}

Die Regelungen zur E-Bilanz sind für steuerbegünstigte Körperschaften nicht ganz einfach. Für die Praxis bietet sich unter Beachtung der vorstehenden Überlegungen derzeit folgendes Prüfungsschema an:

1. Besteht Bilanzierungspflicht nach Handelsrecht, z. B. nach dem Handelsgesetzbuch oder der Pflegebuchführungsverordnung?

2. Wenn nein: keine E-Bilanz-Pflicht. Wenn ja: wird ein steuerpflichtiger wirtschaftlicher Geschäftsbetrieb unterhalten?

3. Wenn nein: keine E-Bilanz-Pflicht. Wenn ja: liegen die Brutto-Einnahmen hieraus unter der Besteuerungsgrenze von jährlich 35.000 Euro?

4. Wenn ja: keine E-Bilanz-Pflicht. Wenn nein: die Regelungen zur EBilanz sind zu beachten. Sofern für den steuerpflichtigen Bereich nur eine Einnahmen-Überschuss-Rechnung erstellt wird, ist elektronisch (ab 2016 erstmals für 2015) nur der Gewinn als Einzelbetrag zu übermitteln.

5. Zusatzfrage, sofern die Grenzen des \141 AO für eine steuerliche Bilanzierungspflicht überschritten sind: Hat das zuständige Finanzamt auf die Verpflichtung zur steuerlichen Bilanzierung hingewiesen?

6. Wenn nein (dies sollte der Regelfall sein): Die Regelungen zur E-Bilanz sind $\mathrm{zu}$ beachten. Sofern für den steuerpflichtigen Bereich nur eine Einnahmen-Überschuss-Rechnung erstellt wird, ist elektronisch (ab 2016 erstmals für 2015) nur der Gewinn als Einzelbetrag zu übermitteln. Wenn ja (dies sollte nur ganz ausnahmsweise der Fall sein): Die Regelungen zur EBilanz sind eigentlich vollumfänglich zu beachten. Allerdings könnte auch in diesen Fällen eine elektronische Übermittlung des steuerpflichtigen Gewinns als Einzelbetrag in Betracht kommen. 